\title{
Leukadherins get a grip on inflammation
}

activating
integrins,
instead of
blocking them,
may be a more
successful
therapeutic
approach

Integrins have essential roles in promoting immune cell recruitment to inflamed tissues and have attracted much interest as potential therapeutic targets. However, the integrin-blocking drugs that are currently available have shown limited clinical success, and several of these drugs have been withdrawn from the market owing to undesirable side effects. Now, Vineet Gupta and colleagues describe a new strategy for targeting integrins that they think might catch on.

Current therapies have focused on blocking integrins or their ligands using specific monoclonal antibodies. Such drugs have been effective in many animal models, but have been less successful in treating human disease. This is thought to be due, in part, to the high degree of molecular blockade that is required to inhibit integrinmediated cell migration ( $>90 \%$ of active integrins must be occupied). Early literature on integrins showed that trapping $\alpha 4 \beta 1$ integrin in a high-affinity state leads to increased cellular adhesion to the vascular endothelium and inhibits eosinophil migration. Therefore, the authors

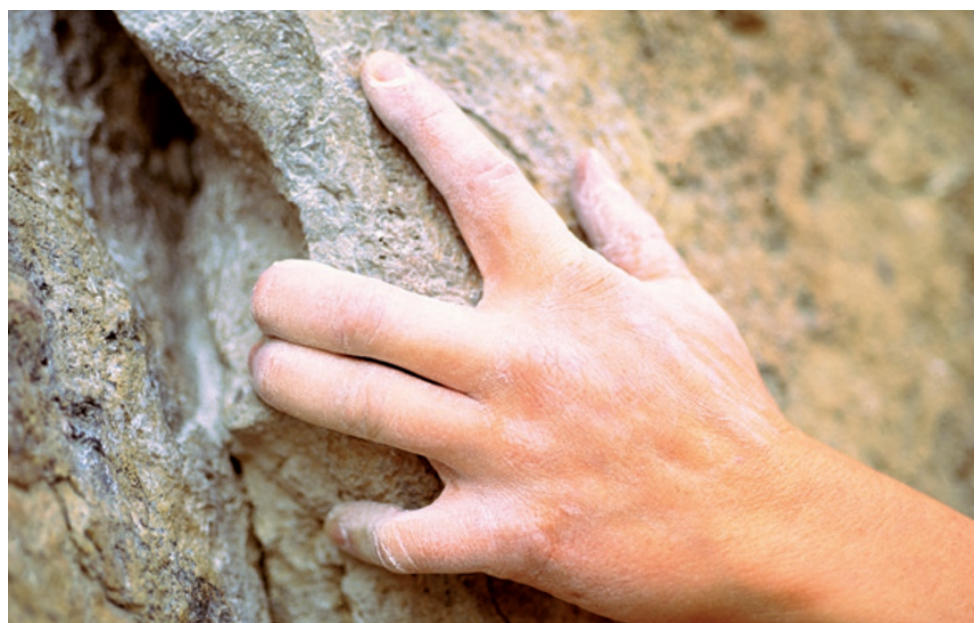

decided to investigate new therapies based on integrin activation rather than blockade.

They had previously used a highthroughput screening assay to find compounds that could affect the adhesion of a cell line expressing $\alpha \mathrm{M} \beta 2$ integrin (also known as CD11bCD18) to fibrinogen. In this way, they identified several $\alpha \mathrm{M} \beta 2$ integrin agonists, which they termed leukadherins. By chemically modifying a core furanyl thiazolidinone structural motif, they further enhanced the ability of leukadherins to promote $\alpha \mathrm{M} \beta 2$ integrin-mediated adhesion in vitro.

The leukadherins were found to promote cell adhesion by activating $\alpha \mathrm{M} \beta 2$ integrin via its ligand-binding $\alpha$ I domain. In live-cell imaging experiments, leukadherins decreased the lateral migration and also the velocity of neutrophils migrating in response to $N$-formyl-methionyl-leucylphenylalanine in vitro. Furthermore, neutrophils exposed to leukadherins had elongated uropods in these assays, suggesting that leukadherins inhibit cell migration by preventing the de-adhesion of cells. In support of this, $\alpha \mathrm{M} \beta 2$ integrin was shown to cluster in the extended uropods of cells treated with leukadherins, and these compounds increased the adhesion of THP1 monocytes to an endothelial cell monolayer in vitro.

The authors next extended their findings in vivo, showing that leukadherins could inhibit the recruitment of neutrophils to the inflamed peritoneum in mice. Leukadherins also reduced macrophage accumulation and limited disease in a rat model of arterial injury. Furthermore, in a mouse model of nephritis, leukadherins were more effective than a well-characterized $\alpha \mathrm{M} \beta 2$ integrinspecific blocking antibody (M1/70) at inhibiting neutrophil infiltration into the kidney and, notably, mice treated with leukadherins developed less severe disease than those treated with M1/70. Intravital imaging of mouse cremaster muscle showed that leukadherins decreased the rolling velocity of leukocytes in tumour necrosis factor-stimulated venules, confirming that these compounds work in vivo by increasing leukocyte adhesion to the endothelium.

The authors' findings are consistent with previous studies, which showed that mice with constitutively active integrin mutants show decreased recruitment of leukocytes to inflamed tissues owing to increased cell adhesion. Importantly, the authors did not see any evidence of systemic toxicity in the rodents that were treated with leukadherins. They therefore suggest that activating integrins, instead of blocking them, may be a more successful therapeutic approach. Yvonne Bordon

ORIGINAL RESEARCH PAPER Maiguel, D. et al. Small molecule-mediated activation of the integrin CD11b/CD18 reduces inflammatory disease. Sci. Signal. 4, ra57 (2011)

FURTHER READING Hogg, N., Patzak, I. \& Willenbrock, F. The insider's guide to leukocyte integrin signalling and function. Nature Rev. Immunol. 11, 416-426 (2011) 